\title{
Evaluation of Clouds and the Earth's Radiant Energy System (CERES) scanner pointing accuracy using a coastline detection system
}

\author{
${ }^{\mathrm{a}}$ Chris Currey, ${ }^{\mathrm{b}}$ Lou Smith, ${ }^{\mathrm{c}}$ Bob Neely \\ ${ }^{a}$ NASA Langley Research Center, Atmospheric Sciences Division \\ Hampton, Virginia 23681 \\ bVirginia Polytechnic Institute and State University, \\ Blacksburg, Virginia 24061 \\ ${ }^{\mathrm{c} S}$ Science Applications International Corporation \\ Hampton, Virginia 23666
}

\begin{abstract}
Clouds and the Earth's Radiant Energy System (CERES) is a National Aeronautics and Space Administration (NASA) investigation to examine the role of clouds in the radiative energy flow through the Earth-atmosphere system. The first CERES scanning radiometer was launched on November 27, 1997 into a $35^{\circ}$ inclination, $350 \mathrm{~km}$ altitude orbit, on the Tropical Rainfall Measuring Mission (TRMM) spacecraft. The CERES instrument consists of a three channel scanning broadband radiometer. The spectral bands measure shortwave $(0.3-5 \mu \mathrm{m})$, window $(8-12 \mu \mathrm{m})$, and total $(0.3-100 \mu \mathrm{m}) \mathrm{radiation}$ reflected or emitted from the Earth-atmosphere system. Each Earth viewing measurement is geolocated to the Earth fixed coordinate system using satellite ephemeris, Earth rotation and geoid, and instrument pointing data. The interactive CERES coastline detection system is used to assess the accuracy of the CERES geolocation process. By analyzing radiative flux gradients at the boundaries of ocean and land masses, the accuracy of the scanner measurement locations may be derived for the CERES/TRMM instrument/satellite system. The resulting CERES measurement location errors are within $10 \%$ of the nadir footprint size. Precise pointing knowledge of the Visible and Infrared Scanner (VIRS) is required for convolution of cloud properties onto the CERES footprint; initial VIRS coastline results are included.
\end{abstract}

Keywords: CERES, VIRS, geolocation, radiometer, validation, coastlines

\section{INTRODUCTION}

Geolocation is the process of calculating the longitude and latitude of a measurement. The determination of the field of view (FOV) centroid location can be separated into two major steps. First, the unit pointing vector of the detector optical axis is calculated in the local horizon system located at the spacecraft. Second, the detector pointing vector is transformed from the local horizon system into Earth fixed coordinates. Details of the CERES geolocation process are described in Ref. 4. Earth Radiation Budget Experiment (ERBE) instrument studies estimated its scanner response time at 46 milliseconds. The scanhead elevation beam rotated at 66.7 degrees/second during the Earth viewing portion of the scan cycle. The scanner elevation angle, which determines the detector pointing vector, was offset $3.066^{\circ}$ to compensate for this time delay. ERBE used a coastline detection algorithm to determine scanner geolocation errors for the Earth Radiation Budget Satellite (ERBS) and NOAA-9 missions. Analysis of November 1984 data using the coastline algorithm detected a small but persistent crosstrack bias of $-.026^{\circ}$ (geographic coordinates). The elevation angle offset was adjusted from $3.066^{\circ}$ to $2.85^{\circ}$ and data reprocessed. ${ }^{1}$

The CERES instrument, ${ }^{2}$ an improved version of the ERBE scanner, consists of a shortwave, window, and total channel coaligned such that they share a $98 \%$ common field of view. Each detector is sampled at $100 \mathrm{~Hz}$ producing approximately $5.86 \times 10^{6}$ Earth-viewing footprints per day. The detectors are mounted on a scanhead assembly which has two degrees of motion: an azimuth drive rotates up to 6 degrees/second, and an elevation drive rotates at 63.5 degrees/second. A scanline 
contains two elevation scans which alternate in direction from Earth horizon to horizon. The instrument has two main scanning modes, cross-track and rotating azimuth. Both azimuth and elevation angles vary in the rotating azimuth mode. Only crosstrack data is analyzed with the coastline technique.

A field stop aperture restricts the sensor optical field of view to $1.3^{\circ}$ in the instrument along-scan direction and $2.6^{\circ}$ in the cross-scan direction. For TRMM, the optical FOV projected onto the Earth's surface at nadir is $8 \mathrm{~km}$ along the scan direction and $16 \mathrm{~km}$ wide. The CERES point spread function (PSF) defines the actual shape of a footprint by accounting for the dynamic response of the detectors and filter electronics. ${ }^{3}$ The $95 \%$ energy point PSF causes the footprint at nadir to grow to $16 \times 16 \mathrm{~km} .{ }^{4}$ The PSF produces a time lag in the output radiance relative to the centroid of the optical field of view. The flight radiance count conversion equation ${ }^{5}$ incorporates $\gamma$, the average time delay between the instantaneous sensor's optical axis and the measurement's point spread function centroid. Simulation and preflight ground calibration measurements determined $\gamma$ to be 23 milliseconds. The ground processing system offsets the elevation angle to account for this time delay. The coastline analysis provides a check of this offset.

Measurements from the TRMM VIRS instrument are used to determine cloud properties within the CERES footprint. VIRS consists of $0.63,1.61,3.75,10.80$, and $12.00 \mu \mathrm{m}$ channels each with $2.11 \mathrm{~km}$ resolution at nadir. Identification of VIRS cross-track or along-track biases will aid in the CERES cloud determination process.

This paper describes the coastline analysis method of assessing scanner measurement geolocation accuracy. This technique should be effective for scanners with footprints greater than several kilometers in size (CERES $\sim 10 \mathrm{~km}$ at nadir). Initial TRMM CERES and VIRS results are presented.

\section{ALGORITHM DESCRIPTION}

The CERES coastline detection system provides several improvements to the ERBE geolocation assessment process.

- Unlimited coastline site selection

- Interactive processing with visualization

- Better clear sky screening

- Better understanding of the PSF effects on geolocation

Coastlines with high thermal gradients, e.g. deserts adjacent to ocean, provide excellent sites for coastline analysis. High thermal measurements are presumed to be land during the day and water at night. Low thermal measurements are presumed to be water during the day and land at night. This reversal in the longwave signature allows processing of both day and night data. ERBE scenes were limited to four desert/ocean geographic sites: Baja, northern Australia, Libya, and Oman. CERES provides coastline analysis of any geographic site using shortwave, window, or total channel data. Scenes are selected through interactive visualizaton. For each coastline scene, an ensemble of detected crossings is collected and compared to a digitized map. Scene location errors are determined by minimizing the average distance between the crossings and the map. Samples are collected to identify systematic biases.

The CERES radiometer measurements are located at the "top of the atmosphere", $30 \mathrm{~km}$ above the WGS-84 ellipsoid model, and at the Earth's surface. The intersection of the instrument pointing vector with the Earth ellipsoid determines the measurement's surface geolocation. The surface geocentric latitude $\phi_{\text {surf }}$ and longitude $\Lambda_{\text {surf }}$ are defined in Earth fixed coordinates as

$$
\begin{aligned}
& \phi_{\text {surf }}=\operatorname{asin}\left(S_{z} /|S|\right) \\
& \Lambda_{\text {surf }}=\operatorname{atan}\left(S_{y} / S_{x}\right)
\end{aligned}
$$

where $\vec{S}$ is the position vector in cartesian coordinates. Geocentric locations are converted to geodetic coordinates by 


$$
\tan \phi=\left(a^{2} / b^{2}\right) \tan \phi^{\prime}
$$

where $\mathrm{a}$ is the Earth semimajor axis $(6378.137 \mathrm{~km}), \mathrm{b}$ is the semiminor axis $(6356.752 \mathrm{~km}), \phi$ is the geodetic latitude, and $\phi$ is the geocentric latitude. The CERES surface geodetic locations are compared to the public domain World Bank II high resolution map which is digitized at approximately $0.2 \mathrm{~km}$ resolution.

The coastline algorithm searches for coastlines by traversing a scanline and fitting four consecutive measurements to a cubic equation $y_{i}=\mathrm{a} x_{i}^{3}+\mathrm{b} x_{i}^{2}+\mathrm{c} x_{i}+\mathrm{d}$, where $y_{i}$ is the radiance and $x_{i}$ is the position of each measurement. The coefficients are solved by

$$
\left[\begin{array}{l}
a \\
b \\
c \\
d
\end{array}\right]=\left[\begin{array}{llll}
x_{1}^{3} & x_{1}^{2} & x_{1} & 1 \\
x_{2}^{3} & x_{2}^{2} & x_{2} & 1 \\
x_{3}^{3} & x_{3}^{2} & x_{3} & 1 \\
x_{4}^{3} & x_{4}^{2} & x_{4} & 1
\end{array}\right]^{-1}\left[\begin{array}{l}
y_{1} \\
y_{2} \\
y_{3} \\
y_{4}
\end{array}\right]
$$

The inflection point, $\mathrm{x}=-\mathrm{b} / 3 \mathrm{a}$, is considered a coastline crossing if it falls between $x_{2}$ and $x_{3}$, it is within a specified distance from the map, and a significant energy delta occurs between $y_{1}$ and $y_{4}$. Figure 1 depicts a typical radiance scan as it crosses a coastline with an inflection point that meets the radiance threshold criteria. Online tuning of the energy threshold allows processing of scenes during different times of the day. Scenes must be cloud free to prevent false coastline detections. CERES scenes are screened for cloud contamination by analyzing higher resolution VIRS data.

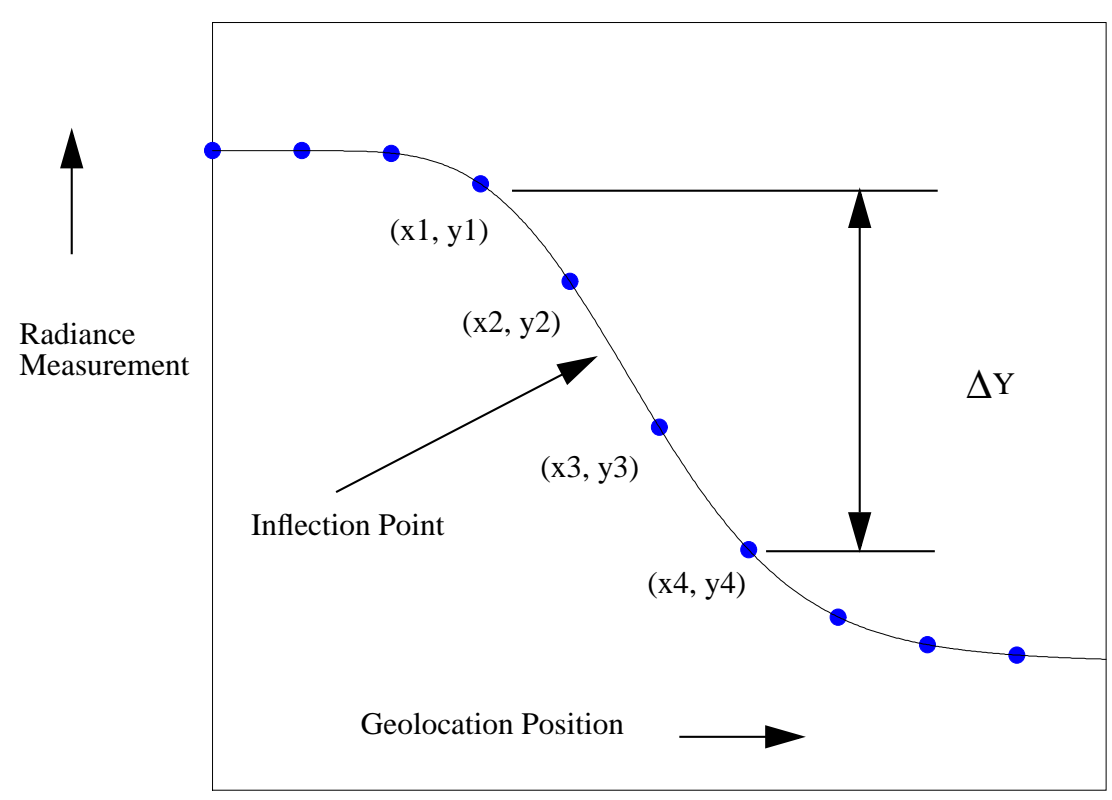

Figure 1. Cubic fit for coastline detection. 
The downhill simplex method ${ }^{6}$ is used to minimize the average distance between the ensemble of crossings and the map database. Translational adjustments are applied to each crossing location (longitude, latitude). The distance function is iteratively calculated until successive solutions fall within an acceptable tolerance. The resulting longitude/latitude shift is the location error for the scene. Since much of the location error may be attributed to the spacecraft attitude or the detector elevation assembly, errors are mapped into a coordinate system aligned with the spacecraft ground track and instrument scan axis. The mapping of errors in geographic coordinates is shown in figure 2 and given by

$$
\left[\begin{array}{l}
\varepsilon_{c} \\
\varepsilon_{a}
\end{array}\right]=\left[\begin{array}{cc}
\mp \sin \eta & \mp \cos \eta \\
\cos \eta & \cos \eta
\end{array}\right]\left[\begin{array}{l}
\varepsilon_{\Lambda} \\
\varepsilon_{\phi}
\end{array}\right]
$$

where $\eta$ is the spacecraft heading angle, $\varepsilon_{c}$ is the cross-track error, $\varepsilon_{a}$ is the along-track error, $\varepsilon_{\Lambda}$ is the longitude error, and $\varepsilon_{\phi}$ is the latitude error. The along-track axis is positive in the direction of spacecraft flight. The cross-track axis is positive in the instrument scan direction. The upper sign (-) in equation (4) is used for left to right scans; the lower sign (+) is used for right to left scans. The coordinate systems, based on scan direction, support spacecraft $+\mathrm{X}$ axis and $-\mathrm{X}$ axis forward.

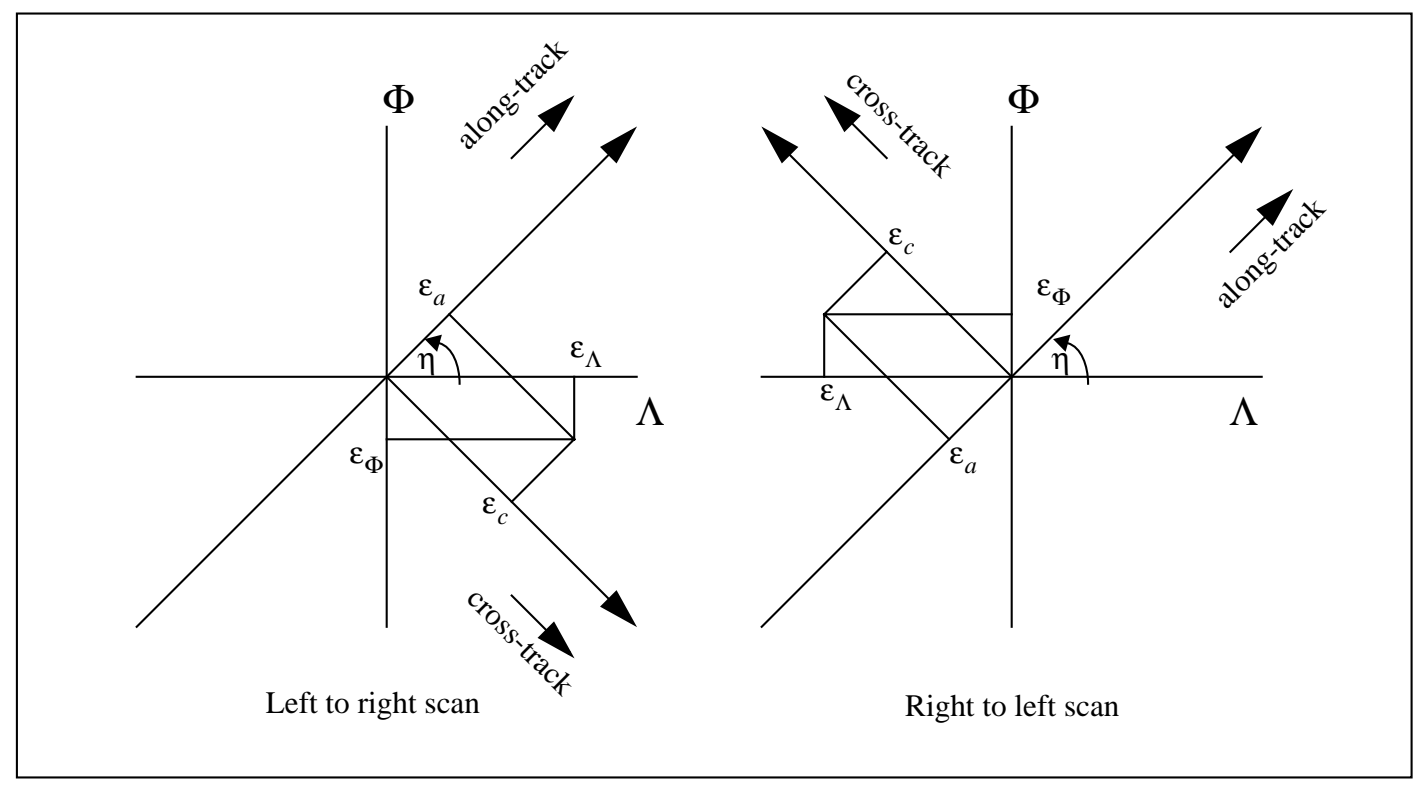

Figure 2. Transformation from Earth fixed coordinate system to along-track cross-track coordinates.

Simulation results using the CERES PSF theoretical model provide error bounds for the coastline technique. The PSF defines the effect of the radiance at each point within the field of view on the measurement. Figure 3 shows the variation of the PSF theoretical model in the along-scan $x$ and cross-scan y directions. The PSF is asymmetric due to the time responses of the detector and electronic filter circuitry. The peak value (diamond) is $1.36^{\circ}$ and the centroid (asterisk) is $1.51^{\circ}$ from the optical axis. The PSF centroid is the desired geolocation point for a footprint; the PSF peak is used to identify a coastline crossing. Coastline results must account for this $0.15^{\circ}$ difference. The coast is considered to be a knife edge, i.e. a step-function change. As the instrument scans over a coast, the measurement is the integral of the part of the PSF over the land:

$$
I_{2}(\xi)=\iint_{\xi \eta} P S F(x, y) d \eta d \xi
$$




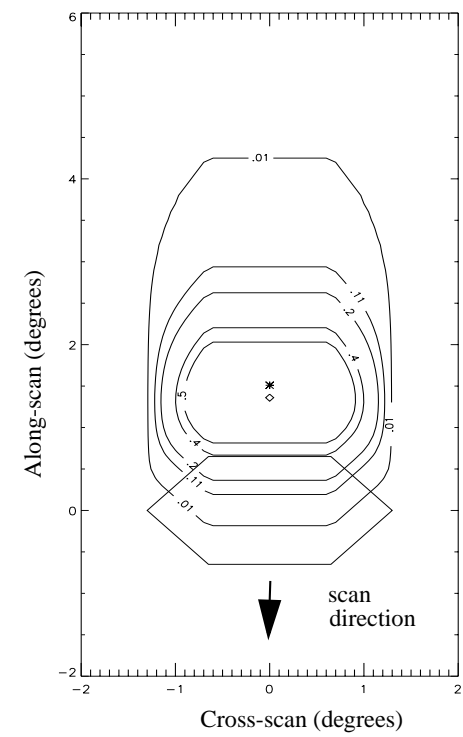

Figure 3. CERES point spread function.

where $\eta$ is the distance parallel to the coast and $\xi$ is the distance normal to the coast. The inflection point of the double integral is at the location of the peak of the first integral. The inflection point calculation provides an accurate measurement of the PSF peak. Random CERES sampling of the PSF step response, 1000 runs at $\theta=0^{\circ}$, produces the inflection point at $1.36^{\circ}(\sigma=$ $\left..02^{\circ}\right)$.

Figure 4 shows the effect of varying the angle between the coast normal and the scan axis on the PSF response and detector output. The angle of the scan direction relative to the normal of the coast is denoted as $\theta$ and varies from $0^{\circ}$ to $45^{\circ}$ in $5^{\circ}$ increments. The optical axis crosses the coast at an along-scan angle of $0^{\circ}$. In figure 4 the PSF response is depicted as the first integral of the PSF function parallel to the coast. The narrowest PSF response is given by the scan normal to the coast for which the peak is at a scan angle of $1.36^{\circ}$. For $\theta \neq 0^{\circ}$, the shoulder of the PSF reaches the coast before the centerline and also leaves the coast later, so that the first integral of the PSF is much wider for $\theta=45^{\circ}$ than for $\theta=0^{\circ}$. The peak of the function moves back with increasing $\theta$, so that for the worst case considered, the peak has moved $.07^{\circ}$ to a scan angle of $1.43^{\circ}$ at $\theta=$ $45^{\circ}$. The PSF centroid remains constant at $1.51^{\circ}$. For uniform sampling of $\theta$ between $0^{\circ}$ and $45^{\circ}$, the average PSF peak shifts $.025^{\circ}$ in the along-scan direction. Coastlines selected for analysis should be relatively perpendicular to the instrument scan axis to reduce variability in the detection process. Understanding the difference between the PSF centroid and peak provides better interpretation of coastline results. 


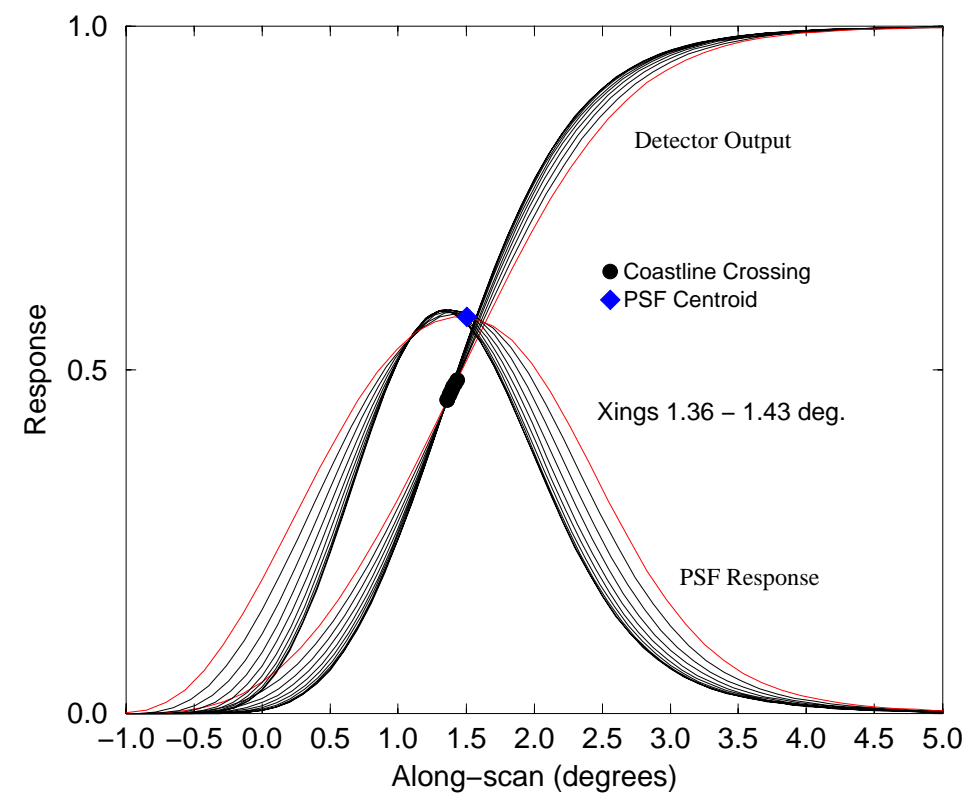

Figure 4. PSF coastline simulation results.

\section{COASTLINE DETECTION SYSTEM DESCRIPTION}

The coastline detection algorithm is embedded in an interactive visualization environment for better scene identification and faster feedback on the geolocation process. Interactive visualization of the shortwave, window, or total channel aids in screening scenes with cloud contamination or improper radiant flux gradients due to diurnal sampling. Online subsetting of a high resolution world map coincident with scene data provides immediate processing of any coastline scene. The World Bank II map database contains almost six million vectors. A special version of the database was created containing coastlines, islands, lakes, and rivers. Each continent contains several thousand varying length polyline segments. Figure 5 shows a typical coastline detection system operating environment. Pop-up windows provide control over sample collection, processing, and archiving of results. For CERES the user searches for clear scenes within a Bidirectional Scans (BDS) data product $(\sim 700 \mathrm{MB})$, a daily data set containing 24 hours of instantaneous measurements. Gross geolocation errors are quickly detected. Scene results are archived in an ASCII file for identification of systematic cross-track and along-track biases. The coastline detection system was developed using the IBM Data Explorer software operating on UNIX workstations. A visual program, or network, is generated by connecting modules using a visual programming editor. Custom modules for importing Hierarchical Data Format (HDF) BDS files, coastline detection, and error minimization are written in the C programming language and linked into the DX network for execution. The network provides scanline plotting, scalar min/max control, subsetting, and filtering by a second parameter. Radiometric channel data may be rendered as points, lines, or smooth shaded. The interactive coastline detection system serves as an excellent development environment for Earth sciences algorithms. New algorithms can be embedded into the network and results visualized in the Earth fixed coordinate system. A separate network, similar to the one for CERES, is used to analyze VIRS data. 


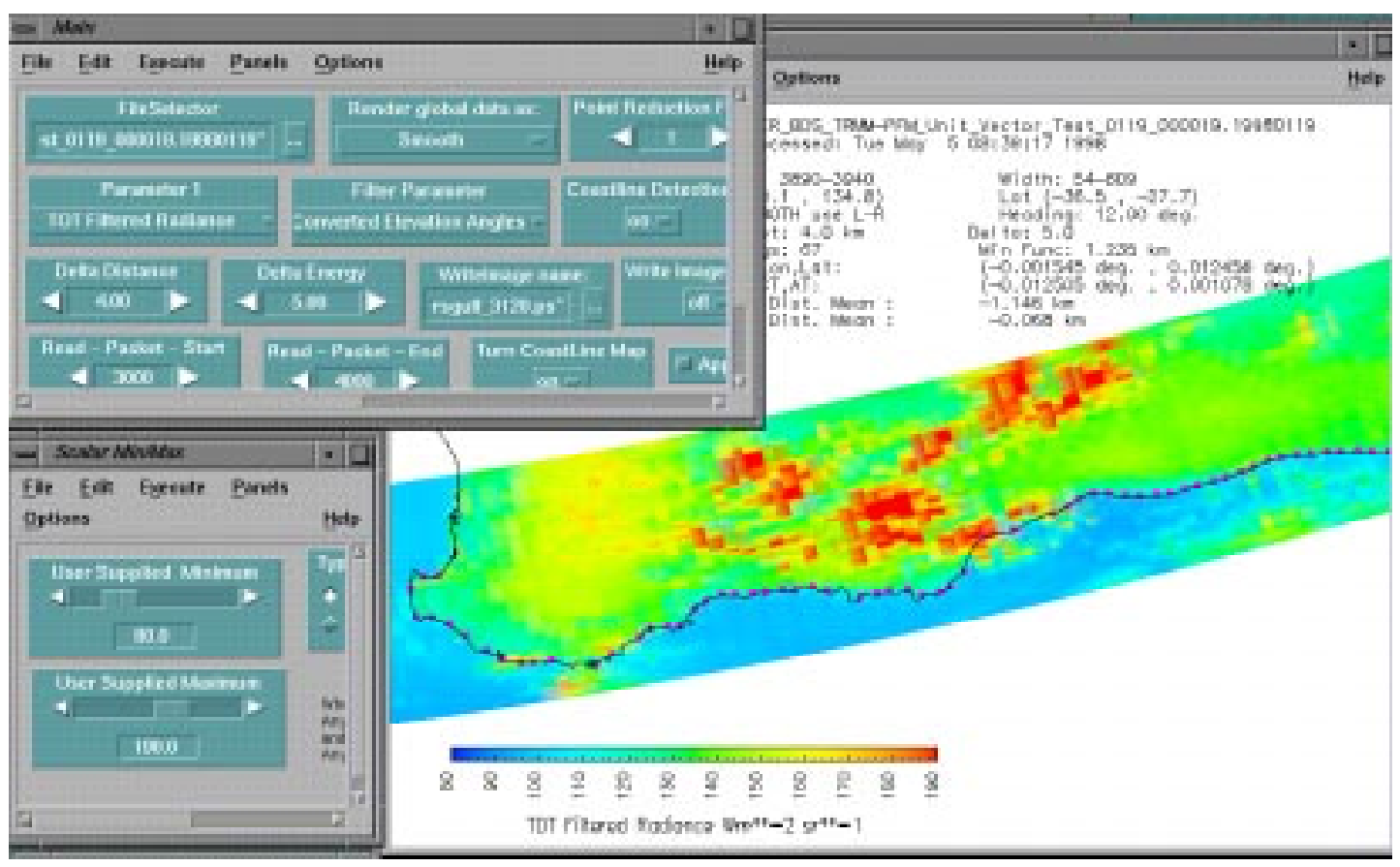

Figure 5. Coastline detection system interactive environment.

\section{TRMM RESULTS AND CONCLUSIONS}

Figure 6 shows a CERES coastline detection sample collected over South Africa on January 16, 1998. Spacecraft heading relative to the equator is $-13^{\circ}$. The minimum total radiance inflection threshold is $10 \mathrm{Wm}^{-2} \mathrm{sr}^{-1}$ and the maximum viewing zenith is $30^{\circ}$. Detected crossings are drawn as circles. The minimization fitting technique calculates a longitude error of $.0098^{\circ}$ and a latitude error of $.0052^{\circ}$. On the Earth's surface, this corresponds to an along-track error of $.92 \mathrm{~km}$ and a crosstrack error of $-.81 \mathrm{~km}$.

Figure 7 shows a VIRS sample collected over the western coast of Mexico on January 3, 1998. Spacecraft heading is $-31.6^{\circ}$ relative to the equator. The minimum brightness temperature threshold is $3 \mathrm{~K}$. The coastline fitting technique minimizes the average distance between the 442 crossings and the digitized map. The resulting longitude error of $-.0008^{\circ}$ and latitude error of.$- .0035^{\circ}$ correspond to an along-track error of $.13 \mathrm{~km}$ and a cross-track error of $.38 \mathrm{~km}$.

The location error for a single coastline scene may not be representative of the instrument/satellite system. Additional satellite passes and different coastlines need to be investigated to identify systematic biases in the along-track cross-track coordinate system. Figure 8 shows CERES results for clear samples collected during January 1998. All samples are filtered for cloud contamination using corresponding VIRS scenes. The average cross-track bias is $-.58 \mathrm{~km}(\sigma=1.11 \mathrm{~km})$; the average alongtrack bias is $.48 \mathrm{~km}(\sigma=1.08 \mathrm{~km})$. Figure 8 also shows the location errors mapped into the instrument coordinate system. The PSF contour of .11 defines the CERES nadir footprint (95\% energy) used in the convolution of imager cloud properties. Average cross-scan bias from the PSF centroid is $.078^{\circ}\left(\sigma=.176^{\circ}\right)$, and average along-scan bias from the PSF centroid is $-.095^{\circ}\left(\sigma=.182^{\circ}\right)$. 


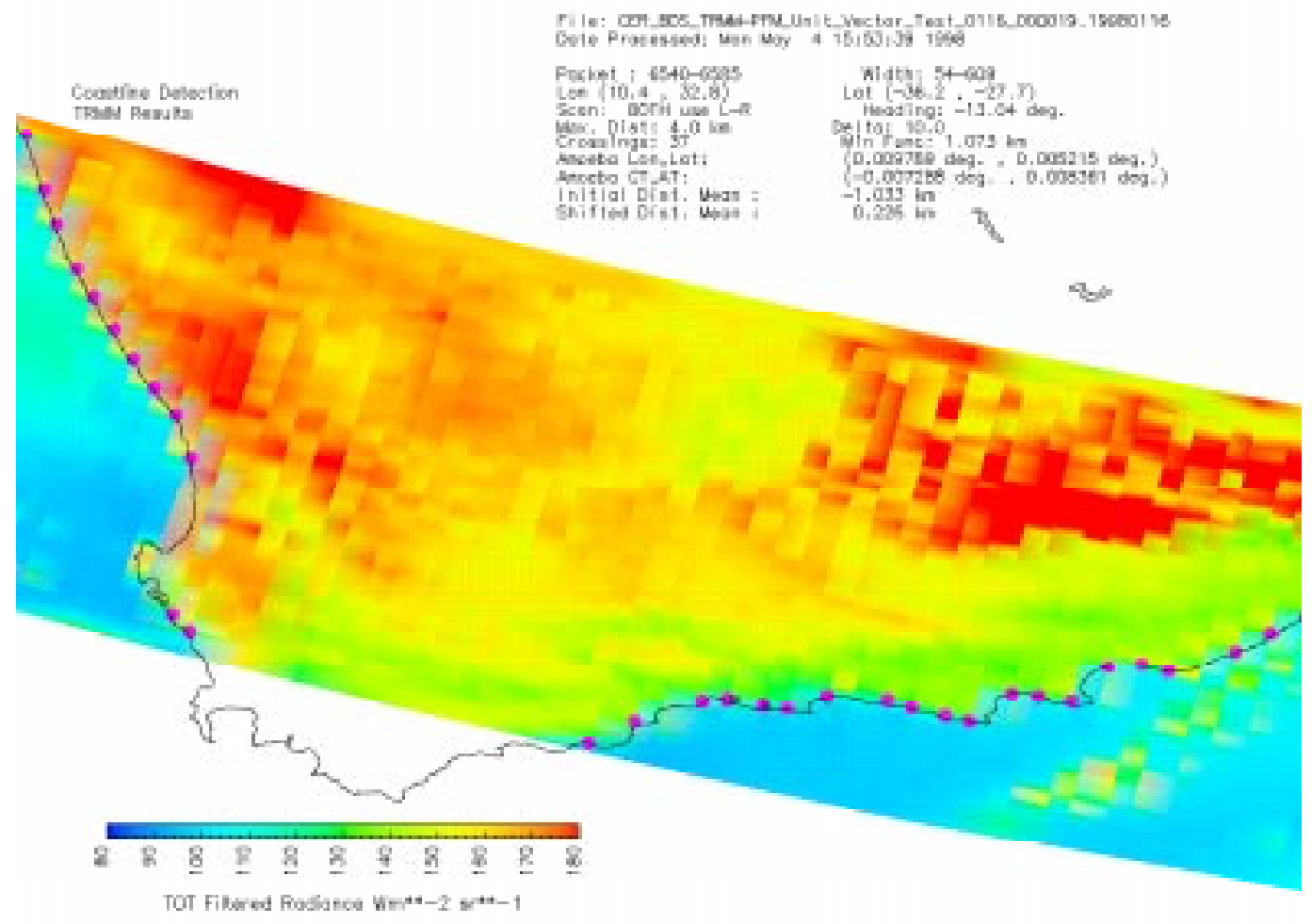

Figure 6. CERES coastline detection sample, South Africa January 16, 1998

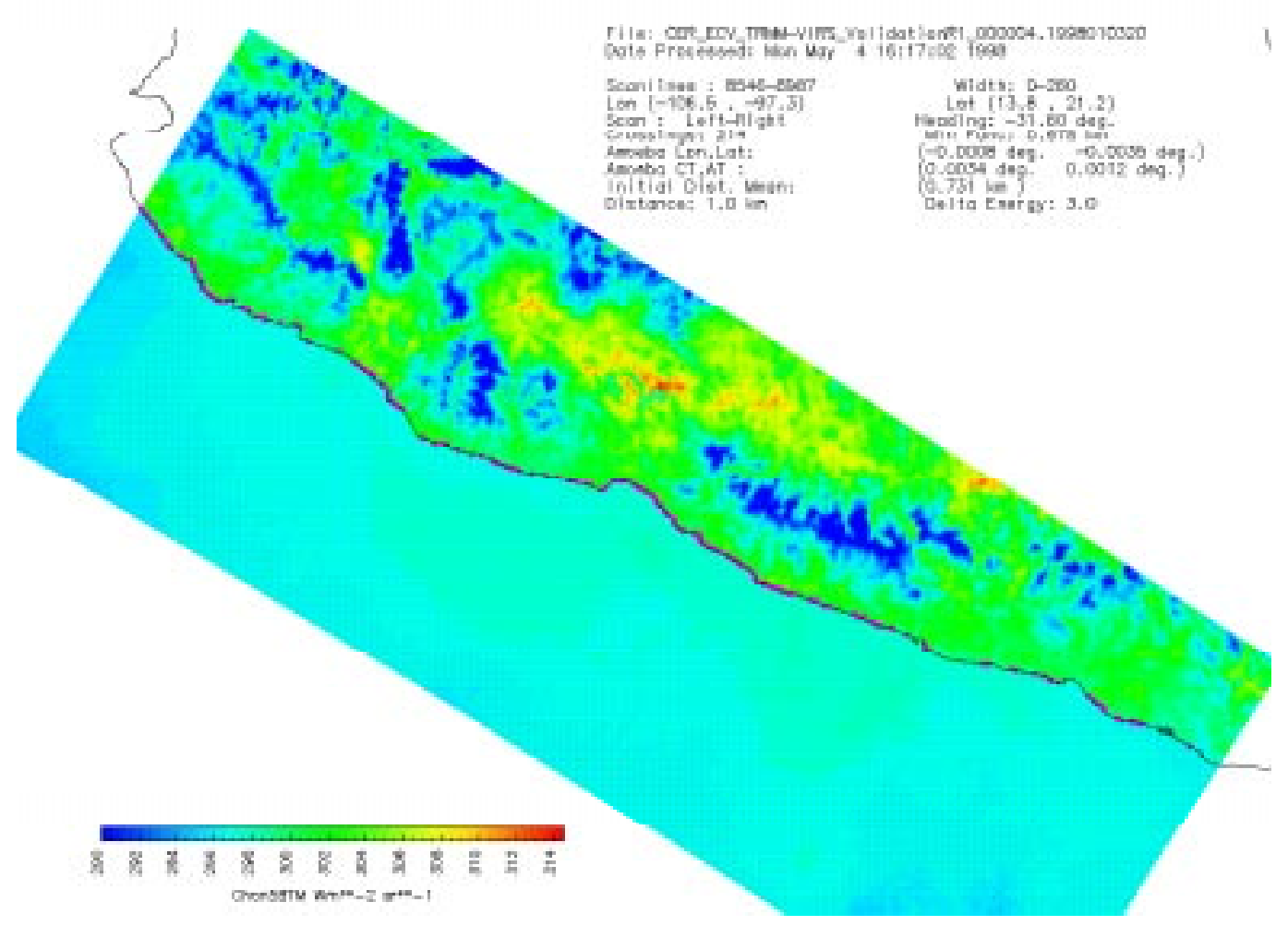

Figure 7. VIRS coastline detection sample, Mexico January 3, 1998 

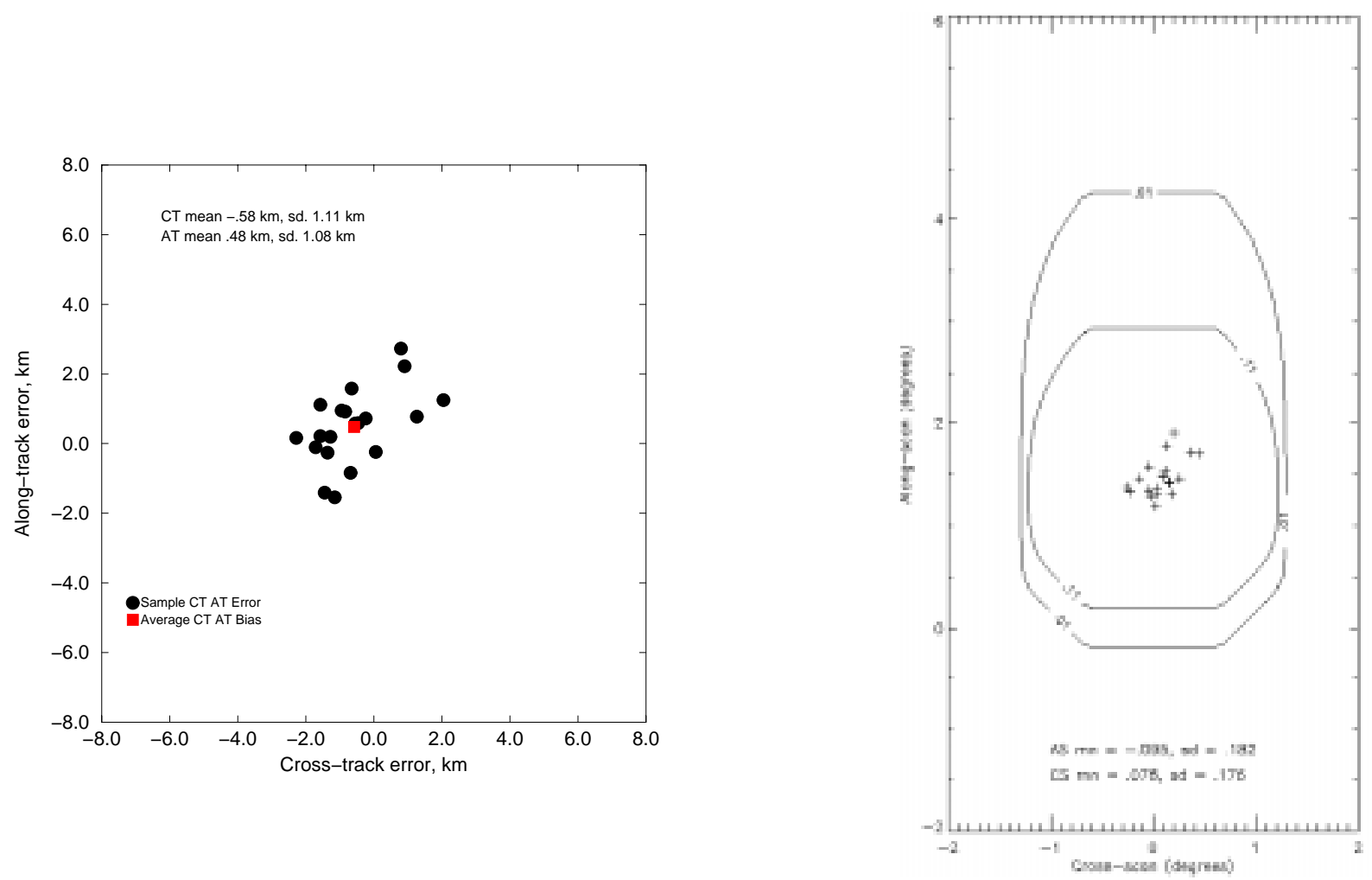

Figure 8. CERES geolocation errors, January 1998, Earth fixed and instrument coordinate systems.

Figure 9 shows results of corresponding VIRS samples for January 1998. The average cross-track bias is .63 km $(\sigma=.29 \mathrm{~km})$; the average along-track bias is $-.20 \mathrm{~km}(\sigma=.46 \mathrm{~km})$. Correspondence with GSFC TSDIS personnel indicate that initial ground processing did not include all VIRS sensor model and alignment parameters. Processing with updated instrument parameters began in April, 1998. April samples processed through the coastline algorithm no longer contain a significant cross-track bias.

The PSF time delay between the optical FOV and centroid affects measurement geolocation. To compensate for this lag, the scanner elevation angle for each footprint is adjusted in the ground processing system by $1.56^{\circ}$. This equates to a $9.5 \mathrm{~km}$ shift of the footprint centroid on the Earth's surface. The coastline detection system provides a gross check of this offset by analyzing forward and backward scans separately. The PSF peak and centroid should differ by $0.15^{\circ}$. If data were perfectly geolocated, and the coastline technique without error, crossings detected from scans of opposite direction would be $1.83 \mathrm{~km}$ apart (peak to peak). Average crossing separation for scenes used in figure 8 is $1.06 \mathrm{~km}$. This results in a $.06^{\circ}$ difference in the ground processing elevation angle offset. Coastline results support the ground studies comparing PSF theory to PSF measurements. ${ }^{7}$

Based upon the results from the coastline detection technique, the accuracy of the CERES scanner measurement location appears to be well within the limits of the detector field of view. The average location error for samples collected in January 1998 is less than $1 \mathrm{~km}$ from the true coastline location; the standard deviation is $1 \mathrm{~km}$ in both the along-track and cross-track directions. Satellite ephemeris and spacecraft attitude appear to be within mission requirements and correctly merged with Level 0 instrument data. The launch environment appears not to have altered preflight conditions. The CERES project will continue to monitor TRMM CERES pointing accuracy with the coastline detection system. Future CERES instruments on the Earth Observing System (EOS) AM and PM satellites will also use the coastline detection technique. 


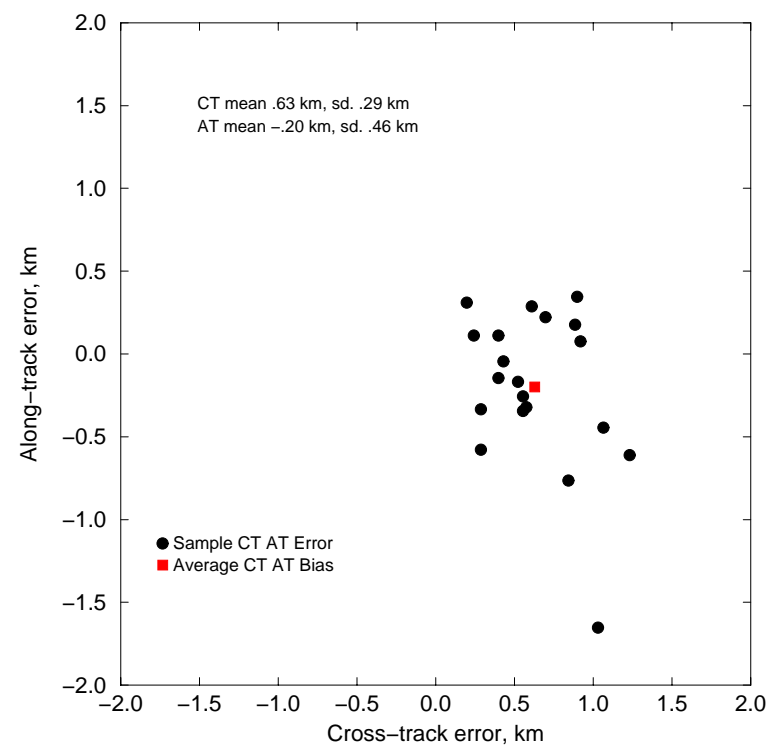

Figure 9. VIRS geolocation errors, January 1998, Earth fixed coordinate system.

\section{ACKNOWLEDGEMENTS}

The authors thank Richard Green and Jim Kibler for their contributions to the algorithm development.

\section{BIBLIOGRAPHY}

1. L. Hoffmann, W. Weaver, and J. Kibler, “Calculation and Accuracy of ERBE Scanner Measurement Locations," NASA Technical Paper 2670, 1987.

2. L. Smith, R. Lee, B. Barkstrom, B. Wielicki, K. Priestley, S. Thomas, J. Paden, R. Wilson, D. Pandey, and K. Thornhill, "Overview of CERES Sensors and In-flight Performance," Proc. of SPIE, Earth Observing Systems III, 1998.

3. L. Smith, "Effects of Time Response on the Point Spread Function of a Scanning Radiometer," Appl. Opt. Vol. 33, No. 30, pp. 7031-7037, 1994.

4. $\quad$ R. Green, B. Wielicki, "Clouds and the Earth's Radiation Energy System (CERES) Algorithm Theoretical Basis Document,” NASA Reference Publication 1376, Volume III, pp. 177-194, 1995.

5. R. Lee, B. Childers, B. Barkstrom, L. Smith, D. Crommelynck, W. Bolden, J. Paden, D. Pandey, S. Thomas, R. Wilson, K. Bush, P. Hess, and W. Weaver, "Clouds and the Earth's Radiation Energy System (CERES) Algorithm Theoretical Basis Document," NASA Reference Publication 1376, Volume II, pp. 1-58, 1995.

6. W. Press, B. Flannery, S. Teukolsky, and W. Vetterling, Numerical Recipes in C, pp. 305-309, Cambridge University Press, Cambridge, 1988.

7. J. Paden, L. Smith, R. Lee, D. Pandey, K. Priestley, H. Bitting, S. Thomas, and R. Wilson, "Comparisons between Point Response Function measurements and theory for the Clouds and the Earth's Radiant Energy System (CERES) TRMM and the EOS-AM spacecraft thermistor bolometer sensors," Proc. of SPIE, Earth Observing Systems III, 1998. 